hep-th/9710090

\title{
Three-Point Spectral Density in QED and the Ward Identity at Finite Temperature
}

\author{
Hou Deful and U. Heinz \\ Institut für Theoretische Physik, Universität Regensburg, \\ D-93040 Regensburg, Germany
}

(October 29, 2018)

\begin{abstract}
We derive the spectral representations of QED 3-point functions and then explicitly calculate the 3-point spectral densities in hard thermal loop approximation within the real time formalism. The Ward identities obeyed by the retarded and advanced 2- and 3-point functions are discussed. We compare our results with those for hot QCD .
\end{abstract}

Typeset using REVTEX 


\section{INTRODUCTION}

Spectral densities are important quantities in finite temperature field theory [1,2]. But they are not easy quantities to evaluate perturbatively at nonzero temperature, especially for many-point spectral densities. The two-point spectral densities have been widely studied and applied to QGP studies [3 9]. In Ref. [7] the Cutkosky rules for calculating the imaginary parts of thermal Green functions using the formalism of Thermo-Field Dynamics (TFD) was presented. Recently these cutting rules were reexamined within the Closed Time Path (CTP) formalism [10,11] and given a simple physical interpretation [12]. The imaginary time cutting rules for calculating two-point spectral densities were investigated in [6]. Threepoint spectral densities, on the other hand, have so far received less quantitative attention. The only available calculation for gauge theories is published in [13 where the 3-point spectral densities for pure gluon dynamics were calculated in the Hard Thermal Loop (HTL) approximation using the imaginary time formalism (ITF) [14]. The spectral representation of three-point functions for selfinteracting scalar fields were discussed in Refs. [15 18].

Hard Thermal Loops (HTLs) are gauge invariant and satisfy simple abelian Ward identities [14]. These remarkable properties have triggered many interesting investigations [19 24]. The computation oh HTLs is generally fairly technical because of their complicated momentum and energy dependence, but it can be simplified by using the Ward identities [14,25]. All HTLs can be derived from a generating functional based on an effective Lagrangian [19]. They describe classical aspects of hot field theories and can thus also be obtained from classical kinetic equations [26,23,24]. However, as Taylor stated in [13], for many purposes one does not need the HTL amplitudes themselves, but only their discontinuities which are described by spectral densities. These spectral densities also provide a natural connection between the Green functions in the real time formulation (RTF) of thermal field theory and

the ITF. While the HTL resummation method was developed within the imaginary time formalism (ITF), with primary attention focussed on equilibrium properties of hot field theories, realistic physical systems are frequently out of thermal equilibrium and require 
calculations in real time. Recently this has motivated increased interest in the real time formulation of thermal field theories and its non-equilibrium extensions.

In the present paper we therefore study the spectral functions and Ward identities for finite temperature QED in the real time formalism (RTF). Extending the recently derived, completely general spectral representation of the real-time 3-point vertex function at finite temperature from scalar field theory to the case of QED, we then show how to implement the HTL approximation in real time. As shown in [27], for 2-point functions this procedure allows for a simple generalization of the HTL resummation scheme to general non-equilibrium situations. Within the HTL approximation we evaluate explicitly the 3-point spectral densities and derive a set of finite temperature Ward identities between the real-time 2- and 3-point functions. Although one needs in general two independent spectral densities to describe the real-time 3-point vertex at finite temperature [17,18, the two are shown to become degenerate in the HTL approximation. This agrees with previous findings in Refs. [13,23. Our Ward identities between the real-time Hard Thermal Loops in QED also agree with the general real-time finite temperature Ward identities recently derived in Ref. 28.].

Throughout this paper we will use the CTP formalism [10] in the form given in Refs. [1],[17]. In this representation of the real-time formalism the bosonic single-particle propagator in momentum space has the form

$$
D(p)=\left(\begin{array}{ll}
D_{11} & D_{12} \\
D_{21} & D_{22}
\end{array}\right)
$$

with

$$
\begin{aligned}
& i D_{11}(p)=\left(i D_{22}\right)^{*}=i \mathcal{P}\left(\frac{1}{p^{2}-m^{2}}\right)+\left(n\left(p_{0}\right)+\frac{1}{2}\right) \rho(p), \\
& i D_{12}(p)=n\left(p_{0}\right) \rho(p) \\
& i D_{21}(p)=\left(1+n\left(p_{0}\right)\right) \rho(p) .
\end{aligned}
$$

Here $n\left(p_{0}\right)$ is the thermal Bose-Einstein distribution,

$$
n\left(p_{0}\right)=\frac{1}{e^{\beta p_{0}}-1}
$$


and $\rho(p)$ is the two-point spectral density which for free particles is given by

$$
\rho(p)=2 \pi \operatorname{sgn}\left(p_{0}\right) \delta\left(p^{2}-m^{2}\right)
$$

The fermionic $2 \times 2$ propagators are given by

$$
S_{a b}(p)=(\not p+m) \tilde{S}_{a b}, \quad(a, b=1,2),
$$

with

$$
\begin{aligned}
& i \tilde{S}_{11}(p)=\left(i \tilde{S}_{22}\right)^{*}=i \mathcal{P}\left(\frac{1}{p^{2}-m^{2}}\right)+\left(-\tilde{n}\left(p_{0}\right)+\frac{1}{2}\right) \rho(p), \\
& i \tilde{S}_{12}(p)=-\tilde{n}\left(p_{0}\right) \rho(p) \\
& i \tilde{S}_{21}(p)=\left(1-\tilde{n}\left(p_{0}\right)\right) \rho(p) .
\end{aligned}
$$

Here $\tilde{n}\left(p_{0}\right)$ is the thermal Fermi-Dirac distribution,

$$
\tilde{n}\left(p_{0}\right)=\frac{1}{e^{\beta p_{0}}+1}
$$

and for free fermions $\rho(p)$ is again given by (4).

The paper is organized as follows. In Sec. [1 we derive the spectral representations for the retarded 3-point functions in QED. In Sec. III we evaluate the 3-point spectral densities for QED in the HTL approximation. In Sec. [V] we derive the RTF Ward identities between the 2- and 3-point HTL amplitudes in QED. A short summary is given in Sec. $\square$.

\section{SPECTRAL REPRESENTATION OF THE 3-POINT VERTEX IN QED}

In this Section we shortly review some useful relations among the different thermal components of the QED 3-point functions and derive their spectral representation. Similar relations for the 3-point vertex in $\phi^{3}$ theory have been reported in the literature [0, 115, 16] in different notation. Our procedure here follows the notation developed in 18 for $\phi^{3}$ theory. We consider the 3-point vertex function in QED shown in Fig. 1. The three incoming external momenta are $k_{1}=p, k_{2}=q$, and $k_{3}=-p-q$. 


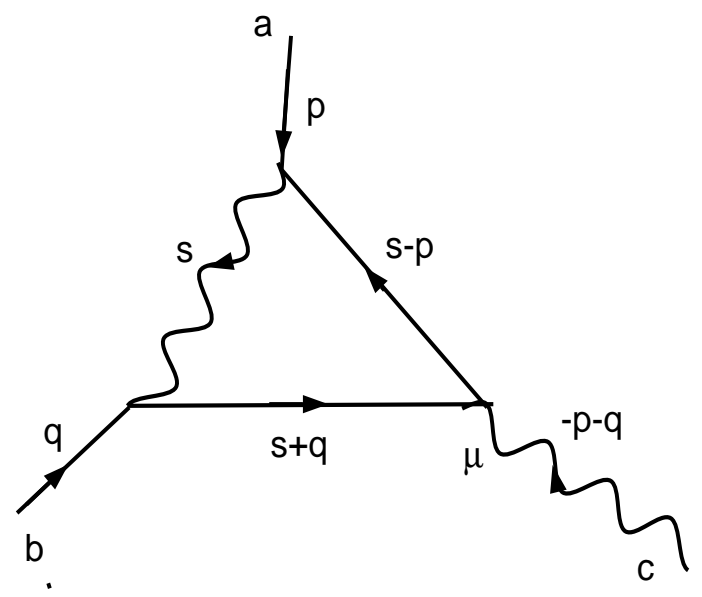

Fig. 1: 3-point vertex in QED

Within the real time formalism, the truncated three-point function for $\mathrm{QED}, G_{a b c}^{\mu}(x, y, z)$, has eight thermal components which satisfy [7, 10]

$$
\sum_{a, b, c=1}^{2} G_{a b c}^{\mu}=0 .
$$

Using the KMS condition one finds in momentum space [30]

$$
\begin{aligned}
& \tilde{G}_{111}^{\mu}\left(k_{1}, k_{2}, k_{3}\right)=-G_{111}^{\mu *}\left(k_{1}, k_{2}, k_{3}\right)=G_{222}^{\mu}\left(k_{1}, k_{2}, k_{3}\right), \\
& \tilde{G}_{121}^{\mu}\left(k_{1}, k_{2}, k_{3}\right)=-G_{121}^{\mu *}\left(k_{1}, k_{2}, k_{3}\right)=-e^{\beta \omega_{2}} G_{212}^{\mu}\left(k_{1}, k_{2}, k_{3}\right), \\
& \tilde{G}_{211}^{\mu}\left(k_{1}, k_{2}, k_{3}\right)=-G_{211}^{\mu *}\left(k_{1}, k_{2}, k_{3}\right)=-e^{\beta \omega_{1}} G_{122}^{\mu}\left(k_{1}, k_{2}, k_{3}\right), \\
& \tilde{G}_{112}^{\mu}\left(k_{1}, k_{2}, k_{3}\right)=-G^{\mu *} 112\left(k_{1}, k_{2}, k_{3}\right)=e^{\beta \omega_{3}} G_{221}^{\mu}\left(k_{1}, k_{2}, k_{3}\right),
\end{aligned}
$$

where $\tilde{G}^{\mu}$ represents "tilde conjugation" of $G^{\mu}$ (see [18]). Note that in Eqs. (9b,9d) the last equation involves an additional minus sign relative to the scalar case [18, due to the fermionic legs.

One can construct "retarded" vertex functions from the above eight thermal components according to

$$
\begin{aligned}
& G_{R}^{\mu}=G_{111}^{\mu}+G_{112}^{\mu}+G_{211}^{\mu}+G_{212}^{\mu}, \\
& G_{R i}^{\mu}=G_{111}^{\mu}+G_{112}^{\mu}+G_{121}^{\mu}+G_{122}^{\mu}, \\
& G_{R o}^{\mu}=G_{111}^{\mu}+G_{121}^{\mu}+G_{211}^{\mu}+G_{221}^{\mu},
\end{aligned}
$$


where $G_{R i}^{\mu}$ is the vertex function which in coordinate space is retarded with respect to $x_{0}$, $G_{R o}^{\mu}$ is retarded with respect to $z_{0}$, and $G_{R}^{\mu}$ is retarded with respect to $y_{0}$. The legs linked to $x, y, z$ correspond to an electron, electron, and photon, respectively, and the corresponding distribution functions are $\tilde{n}_{1}=\tilde{n}\left(\omega_{1}\right), \tilde{n}_{2}=\tilde{n}\left(\omega_{2}\right)$, and $n_{3}=n\left(\omega_{3}\right)$. Inversion of Eqs. (10) with the help of (8) (9) yields expressions for the thermal components $G_{a b c}^{\mu}$ in terms the above retarded functions:

$$
\begin{aligned}
G^{\mu}\left(k_{1}, k_{2}, k_{3}\right) & =G_{R}^{\mu}\left(\begin{array}{c}
\tilde{n}_{1} \\
1-\tilde{n}_{1}
\end{array}\right)\left(\begin{array}{c}
1 \\
-1
\end{array}\right)\left(\begin{array}{c}
-n_{3} \\
1+n_{3}
\end{array}\right)-\frac{1}{2} G_{R}^{\mu *}\left(N_{1}+N_{3}\right)\left(\begin{array}{c}
1 \\
-1
\end{array}\right)\left(\begin{array}{c}
\tilde{n}_{2} \\
1-\tilde{n}_{2}
\end{array}\right)\left(\begin{array}{c}
1 \\
-1
\end{array}\right) \\
& +G_{R i}^{\mu}\left(\begin{array}{c}
1 \\
-1
\end{array}\right)\left(\begin{array}{c}
\tilde{n}_{2} \\
1-\tilde{n}_{2}
\end{array}\right)\left(\begin{array}{c}
-n_{3} \\
1+n_{3}
\end{array}\right)-\frac{1}{2} G_{R i}^{\mu *}\left(N_{2}+N_{3}\right)\left(\begin{array}{c}
\tilde{n}_{1} \\
1-\tilde{n}_{1}
\end{array}\right)\left(\begin{array}{c}
1 \\
-1
\end{array}\right)\left(\begin{array}{c}
1 \\
-1
\end{array}\right) \\
& +G_{R o}^{\mu}\left(\begin{array}{c}
\tilde{n}_{1} \\
1-\tilde{n}_{1}
\end{array}\right)\left(\begin{array}{c}
\tilde{n}_{2} \\
1-\tilde{n}_{2}
\end{array}\right)\left(\begin{array}{c}
1 \\
-1
\end{array}\right)-\frac{1}{2} G_{R o}^{\mu *}\left(N_{1}+N_{2}\right)\left(\begin{array}{c}
1 \\
-1
\end{array}\right)\left(\begin{array}{c}
1 \\
-1
\end{array}\right)\left(\begin{array}{c}
-n_{3} \\
1+n_{3}
\end{array}\right),
\end{aligned}
$$

with $N_{1}=1-2 \tilde{n}_{1}, N_{2}=1-2 \tilde{n}_{2}$, and $N_{3}=1+2 n_{3}$. The structure of this equation is similar to Eq. (35) in Ref. [17]; the sign differences arise from additional minus signs in front of the Fermi distributions and from the fact that Eq. (11) refers to the truncated vertex rather than the connected vertex studied in [17].

Following the same procedure as in Appendix A2 of Ref. 18 one derives the following spectral integral representation for the retarded 3-point functions in QED:

$$
\begin{aligned}
G_{R}^{\mu}\left(\omega_{1}, \omega_{2}, \omega_{3}\right) & =\frac{-i}{2 \pi^{2}} \int_{-\infty}^{\infty} \frac{d \Omega_{1} d \Omega_{2}}{\omega_{2}-\Omega_{2}+i \epsilon}\left(\frac{\rho_{1}^{\mu}}{\omega_{1}-\Omega_{1}-i \epsilon}+\frac{\rho_{1}^{\mu}-\rho_{2}^{\mu}}{\omega_{3}-\Omega_{3}-i \epsilon}\right), \\
G_{R i}^{\mu}\left(\omega_{1}, \omega_{2}, \omega_{3}\right) & =\frac{-i}{2 \pi^{2}} \int_{-\infty}^{\infty} \frac{d \Omega_{1} d \Omega_{2}}{\omega_{1}-\Omega_{1}+i \epsilon}\left(\frac{\rho_{2}^{\mu}}{\omega_{2}-\Omega_{2}-i \epsilon}-\frac{\rho_{1}^{\mu}-\rho_{2}^{\mu}}{\omega_{3}-\Omega_{3}-i \epsilon}\right), \\
G_{R o}^{\mu}\left(\omega_{1}, \omega_{2}, \omega_{3}\right) & =\frac{-i}{2 \pi^{2}} \int_{-\infty}^{\infty} \frac{d \Omega_{1} d \Omega_{2}}{\omega_{3}-\Omega_{3}+i \epsilon}\left(\frac{\rho_{1}^{\mu}}{\omega_{1}-\Omega_{1}-i \epsilon}+\frac{\rho_{2}^{\mu}}{\omega_{2}-\Omega_{2}-i \epsilon}\right) .
\end{aligned}
$$

Here $\omega_{1}+\omega_{2}+\omega_{3}=\Omega_{1}+\Omega_{2}+\Omega_{3}=0$ and the spatial momenta $\boldsymbol{p}_{1}, \boldsymbol{p}_{2}, \boldsymbol{p}_{3}=-\left(\boldsymbol{p}_{1}-\boldsymbol{p}_{2}\right)$ are the same on both sides and have therefore been suppressed. The spectral densities are given by the following thermal components of the 3-point vertex in momentum space:

$$
\begin{aligned}
& \rho_{1}^{\mu}=\operatorname{Im}\left(G_{122}^{\mu}-G_{211}^{\mu}\right), \\
& \rho_{2}^{\mu}=\operatorname{Im}\left(G_{212}^{\mu}-G_{121}^{\mu}\right) .
\end{aligned}
$$


One notes that the spectral representations for the truncated QED three-point functions have the same form as in scalar $\phi^{3}$ theory [18], except for the additional vector index.

\section{EVALUATION OF THE SPECTRAL DENSITIES IN HTL APPROXIMATION}

In this Section we calculate the three-point spectral functions $\rho_{1}^{\mu}, \rho_{2}^{\mu}$ for QED in the hard thermal loop approximation [14. Since HTLs are gauge invariant we can choose Feynman gauge for simplicity.

From Eqs. (9) and (13) we have

$$
\rho_{1}^{\mu}=\operatorname{Im}\left(G_{122}^{\mu}-e^{\beta p_{0}} G_{122}^{* \mu}\right)=\frac{1}{\tilde{n}\left(p_{0}\right)} \operatorname{Im} G_{122}^{\mu}
$$

For $\rho_{1}^{\mu}$ we thus must evaluate only the single Feynman diagram in Fig. 1 for $a=1, b=c=2$. Using standard real-time Feynman rules [10 and the photon propagator in Feynman gauge one gets

$$
G_{122}^{\mu}(p, q,-p-q)=(-i g)(i g)^{2} \int \frac{d^{4} s}{(2 \pi)^{4}}\left[i D_{12}(s)\right] \gamma_{\alpha}\left[i S_{22}(s+q)\right] \gamma^{\mu}\left[i S_{21}(s-p)\right] \gamma^{\alpha}
$$

Inserting the thermal free propagators (2)-(7) and extracting the imaginary part one finds

$$
\begin{aligned}
\operatorname{Im} G_{122}^{\mu}=- & g^{3} \int \frac{d^{4} s}{(2 \pi)^{4}}\left[\gamma_{\alpha}(\not s-\not p+m) \gamma^{\mu}(\not p+\not q+m) \gamma^{\alpha}\right] \\
& \times \delta\left(s^{2}\right) \delta\left((s+q)^{2}-m^{2}\right) \delta\left((s-p)^{2}-m^{2}\right) \operatorname{sgn}\left(s_{0}\right) \operatorname{sgn}\left(s_{0}+q_{0}\right) \operatorname{sgn}\left(s_{0}-p_{0}\right) \\
& \times n\left(s_{0}\right)\left(\frac{1}{2}-\tilde{n}\left(s_{0}+q_{0}\right)\right)\left(1-\tilde{n}\left(s_{0}-p_{0}\right)\right)
\end{aligned}
$$

If the coupling constant $g$ is small and the external momenta are soft, $p, q \sim g T$, and the electron bare mass $m$ is much smaller than the temperature, the leading contributions comes from the hard loop momenta $s \sim T$ [4.14]. For these we can thus neglect the external momenta and the mass $m$ in the terms between square brackets, approximating them by $\gamma_{\alpha} \$ \gamma_{\mu} \phi \gamma_{\alpha}=-4 s_{\mu} \phi+2 \gamma_{\mu} s^{2}$. After performing the integration over $s^{0}$ with the help of the function $\delta\left(s^{2}\right)=\left[\delta\left(s_{0}-\bar{s}\right)+\delta\left(s_{0}+\bar{s}\right)\right] / 2 \bar{s}$, where $\bar{s}=\sqrt{\boldsymbol{s}^{2}}$, one finds 


$$
\begin{aligned}
& \rho_{1}^{\mu}(p, q,-p-q)= \frac{g^{3}}{n\left(p_{0}\right)}\left(A^{\mu}(p, q)+B^{\mu}(p, q)\right) \\
& A^{\mu}(p, q)=\left.\int \frac{d^{3} s}{(2 \pi)} \frac{1}{2 \bar{s}} \operatorname{sgn}\left(\bar{s}+q_{0}\right) \operatorname{sgn}\left(\bar{s}-p_{0}\right) 4 s^{\mu} \not\right|_{s^{0}=\bar{s}} \\
& \times \delta\left(\left(\bar{s}+q_{0}\right)^{2}-E_{s+q}^{2}\right) \delta\left(\left(\bar{s}-p_{0}\right)^{2}-E_{s-p}^{2}\right) \\
& \quad \times n\left(E_{s}\right)\left(\frac{1}{2}-\tilde{n}\left(\bar{s}+q_{0}\right)\right)\left(1-\tilde{n}\left(\bar{s}-p_{0}\right)\right), \\
& B^{\mu}(p, q)=-\left.\int \frac{d^{n-1} s}{(2 \pi)^{n-3}} \frac{1}{2 \bar{s}} \operatorname{sgn}\left(\bar{s}-q_{0}\right) \operatorname{sgn}\left(\bar{s}+p_{0}\right) 4 s^{\mu} \not\right|_{s^{0}=-\bar{s}} \\
& \times \delta\left(\left(\bar{s}-q_{0}\right)^{2}-E_{s+q}^{2}\right) \delta\left(\left(\bar{s}+p_{0}\right)^{2}-E_{s-p}^{2}\right) \\
& \times(n(-\bar{s}))\left(\frac{1}{2}-\tilde{n}\left(-\bar{s}+q_{0}\right)\right) \tilde{n}\left(\bar{s}+p_{0}\right) .
\end{aligned}
$$

Here $E_{s+q}=\sqrt{m^{2}+(\boldsymbol{s}+\boldsymbol{q})^{2}}, E_{s-p}=\sqrt{m^{2}+(\boldsymbol{s}-\boldsymbol{p})^{2}}$, and in (17d) we used the identity $n(-x)+n(x)=-\eta, \eta= \pm 1$ for bosons and fermions.

To simplify the notation it is convenient to introduce the 4 -vectors $V_{ \pm}=\left(1, \pm \frac{s}{s}\right)$. For hard loop momenta the arguments of the $\delta$-functions in (17) can then be written as

$$
\begin{aligned}
& \left.(s+q)^{2}\right|_{s^{0}= \pm \bar{s}} \approx \pm 2 \bar{s} q \cdot V_{ \pm}= \pm 2 \bar{s}\left(q^{0} \mp|\boldsymbol{q}| \cos \theta^{\prime}\right) \\
& \left.(s-p)^{2}\right|_{s^{0}= \pm \bar{s}} \approx \mp 2 \bar{s} p \cdot V_{ \pm}=\mp 2 \bar{s}\left(p^{0} \mp|\boldsymbol{p}| \cos \theta\right),
\end{aligned}
$$

and we can replace $\operatorname{sgn}\left(\bar{s} \pm p^{0}\right) \approx 1 \approx \operatorname{sgn}\left(\bar{s} \pm q^{0}\right)$ under the integral. $\theta$ and $\theta^{\prime}$ are the angles between $\boldsymbol{s}$ and $\boldsymbol{p}, \boldsymbol{q}$, respectively. These approximations decouple the angular and radial integrations [14; we obtain

$$
\begin{aligned}
A^{\mu}(p, q) & \approx a\left(p_{0}\right) \omega^{\mu}(p, q) \\
B^{\mu}(p, q) & \approx b\left(p_{0}\right) \omega^{\mu}(p, q) \\
a\left(p_{0}\right) & =\frac{1}{2 \pi} \int_{0}^{\infty} d \bar{s} \frac{4 \bar{s}^{4}}{(2 \bar{s})^{3}} n(\bar{s})\left(\frac{1}{2}-\tilde{n}\left(\bar{s}+q_{0}\right)\right)\left(1-\tilde{n}\left(\bar{s}-p_{0}\right)\right) \\
b\left(p_{0}\right) & \left.=-\frac{1}{2 \pi} \int_{0}^{\infty} d \bar{s} \frac{4 \bar{s}^{4}}{(2 \bar{s})^{3}} n(-\bar{s})\left(\frac{1}{2}-\tilde{n}\left(-\bar{s}+q_{0}\right)\right) \tilde{n}\left(\bar{s}+p_{0}\right)\right) \\
\omega^{\mu}(p, q) & =\int d \Omega V_{+}^{\mu} V_{+} \delta\left(q \cdot V_{+}\right) \delta\left(p \cdot V_{+}\right)=\int d \Omega V_{-}^{\mu} V_{-} \delta\left(q \cdot V_{-}\right) \delta\left(p \cdot V_{-}\right) .
\end{aligned}
$$

Except for the additional spinor structure the angular integral (198) is identical with the one found by Taylor [13] for the spectral density of the 3-gluon vertex in hot QCD. 
The integrands in Eqs. (19d.19d) contain up to three powers of thermal distribution functions. By using following identities

$$
\begin{aligned}
& n\left(\omega_{1}\right) \tilde{n}\left(\omega_{2}\right)=\tilde{n}\left(\omega_{1}+\omega_{2}\right)\left(1+n\left(\omega_{1}\right)-\tilde{n}\left(\omega_{2}\right)\right), \\
& \tilde{n}\left(\omega_{1}\right) \tilde{n}\left(\omega_{2}\right)=n\left(\omega_{1}+\omega_{2}\right)\left(1-\tilde{n}\left(\omega_{1}\right)-\tilde{n}\left(\omega_{2}\right)\right),
\end{aligned}
$$

one shows that the cubic terms disappear and that $a$ and $b$ reduce to

$$
\begin{aligned}
a\left(p_{0}\right)= & \frac{1}{4 \pi} \int_{0}^{\infty} d \bar{s} \bar{s}\left(\frac{1}{2} \tilde{n}\left(p_{0}\right)\left[n(\bar{s})+\tilde{n}\left(\bar{s}-p_{0}\right)\right]\right. \\
& \left.-n\left(p_{0}+q_{0}\right)\left[\tilde{n}\left(-q_{0}\right)\left[n(\bar{s})+\tilde{n}\left(\bar{s}+q_{0}\right)\right]-\tilde{n}\left(p_{0}\right)\left[n(\bar{s})+\tilde{n}\left(\bar{s}-p_{0}\right)\right]\right]\right), \\
b\left(p_{0}\right)= & \frac{1}{4 \pi} \int_{0}^{\infty} d \bar{s} \bar{s}\left(\frac{1}{2} \tilde{n}\left(p_{0}\right)\left[n(\bar{s})+\tilde{n}\left(\bar{s}+p_{0}\right)\right]\right. \\
& \left.-n\left(p_{0}+q_{0}\right)\left[\tilde{n}\left(-q_{0}\right)\left[n(\bar{s})+\tilde{n}\left(\bar{s}-q_{0}\right)\right]-\tilde{n}\left(p_{0}\right)\left[n(\bar{s})+\tilde{n}\left(\bar{s}+p_{0}\right)\right]\right]\right),
\end{aligned}
$$

The remaining integrands are linear in the $\bar{s}$-dependent thermal distribution functions 31, 18.

After evaluating the integral over $\bar{s}$ in the limit $p_{0} / T \ll 1, q_{0} / T \ll 1$ we obtain to leading order in the coupling constant $g$

$$
\begin{aligned}
\rho_{1}^{\mu}(p, q) & =\frac{g^{3}}{\tilde{n}\left(p_{0}\right)}\left(A^{\mu}(p, q)+B^{\mu}(p, q)\right) \\
& \approx \frac{g m_{\beta}^{2} \pi}{2} \int d \Omega V_{+}^{\mu} V_{+} \delta\left(q \cdot V_{+}\right) \delta\left(p \cdot V_{+}\right) \\
& =\frac{g m_{\beta}^{2} \pi}{2} \int d \Omega V_{-}^{\mu} V_{-} \delta\left(q \cdot V_{-}\right) \delta\left(p \cdot V_{-}\right) .
\end{aligned}
$$

Here $m_{\beta}=\frac{g T}{\sqrt{8}}$ is the thermal electron mass.

The other spectral density $\rho_{2}$ is determined from

$$
\rho_{2}^{\mu}=\frac{1}{\tilde{n}\left(q_{0}\right)} \operatorname{Im} G_{212}^{\mu}
$$

By inspection of the corresponding labelling of the diagram in Fig. 1 one observes that $G_{212}(p, q,-p-q)$ is obtained from $G_{122}(p, q,-p-q)$ by exchanging the two electron legs with the external momenta $p$ and $q$ and routing the internal momentum $s$ in the opposite direction. The resulting loop integral then becomes identical to the one before, Eq. (15), and we find 


$$
\begin{aligned}
\rho_{2}^{\mu}(p, q) & \approx \rho_{1}^{\mu}(q, p) \approx \rho_{\mathrm{HTL}}^{\mu}(p, q), \\
\rho_{\mathrm{HTL}}^{\mu}(p, q) & \approx \frac{g m_{\beta}^{2} \pi}{2} \int d \Omega V_{+}^{\mu} V_{+} \delta\left(p \cdot V_{+}\right) \delta\left(q \cdot V_{+}\right) .
\end{aligned}
$$

This agrees with the observation by Taylor within [13] that in QCD in HTL approximation the two independent spectral densities for the 3-gluon vertex degenerate.

Considering the $\delta$-functions in Eq. (24) one easily shows that

$$
p_{\mu} \rho_{\mathrm{HTL}}^{\mu}(p, q)=0=q_{\mu} \rho_{\mathrm{HTL}}^{\mu}(p, q)
$$

i.e. in HTL approximation the QED spectral density is transverse with respect to the external momenta. This nice feature was also noted in 13 for hot QCD.

\section{REAL-TIME WARD INDENTITIES AMONG HARD THERMAL LOOPS}

Let us insert the above spectral densities into the spectral representations for the retarded 3-point functions:

$$
\begin{aligned}
G_{R}^{\mu}\left(\omega_{1}, \omega_{2}, \omega_{3}\right) & =-i g \frac{m_{\beta}^{2}}{4 \pi} \int d \Omega \frac{V_{+}^{\mu} V_{+}}{\left(V_{+} \cdot k_{1}-i \epsilon\right)\left(V_{+} \cdot k_{2}+i \epsilon\right)}, \\
G_{R i}^{\mu}\left(\omega_{1}, \omega_{2}, \omega_{3}\right) & =-i g \frac{m_{\beta}^{2}}{4 \pi} \int d \Omega \frac{V_{+}^{\mu} V_{+}}{\left(V_{+} \cdot k_{1}+i \epsilon\right)\left(V_{+} \cdot k_{2}-i \epsilon\right)}, \\
G_{R o}^{\mu}\left(\omega_{1}, \omega_{2}, \omega_{3}\right) & =i g \frac{m_{\beta}^{2}}{4 \pi} \int d \Omega \frac{V_{+}^{\mu} V_{+}}{\left(V_{+} \cdot k_{1}-i \epsilon\right)\left(V_{+} \cdot k_{2}-i \epsilon\right)} .
\end{aligned}
$$

The QCD-analogue of last two expressions were previously obtained by Blaizot and Jancu [23] from a set of classical kinetic equations. If the external momenta are of order $g T$, $p_{1} \sim p_{2} \sim g T$, power counting reveals that all three retarded 3-point functions are of order $g$, i.e. of the same order as tree vertex. The HTLs (26) for the QED 3-point vertex in the real time formalism thus require resummation within perturbation theory, like the corresponding HTLs in the imaginary time formalism [14,5].

In the next step we derive the real-time analogue of the well-known Ward identities between the HTL amplitudes in the ITF formalism [25,19]. Due to the matrix structure of the real-time thermal Green functions, the Ward identities also become matrix equations. 
The zero temperature Ward identities for QED can be written both in differential and in integrated form [29]:

$$
\frac{\partial S(p)}{\partial p^{\mu}}=\frac{i}{g} S(p) \Gamma_{\mu}(p,-p, 0) S(p)
$$

or

$$
g\left(S\left(p^{\prime}\right)-S(p)\right)=i S(p)\left[\left(p^{\prime}-p\right)^{\mu} \Gamma_{\mu}\left(p,-p^{\prime}, p^{\prime}-p\right)\right] S\left(p^{\prime}\right) .
$$

Multiplying by $S^{-1}(p)$ from the left and by $S^{-1}\left(p^{\prime}\right)$ from the right and using the SchwingerDyson equation

$$
S^{-1}(p)=\not p-m-\Sigma(p),
$$

the Ward identity takes the form

$$
\left(p^{\prime}-p\right)^{\mu} G_{\mu}\left(p,-p^{\prime}, p^{\prime}-p\right)=-i g\left[\Sigma\left(p^{\prime}\right)-\Sigma(p)\right]
$$

where $G_{\mu}$ is the vertex correction from loop diagrams, defined by $G_{\mu}=\Gamma_{\mu}-\Gamma_{\mu}^{0}$, with $\Gamma_{\mu}^{0}=i g \gamma_{\mu}$

At finite temperature the propagators and vertices have matrix structure. The generalization of (30) to finite temperature in the real time formalism was recently performed in Ref. [28]. The authors of this paper used a different version of the real time formalism and considered the vertex with the photon attached to the ingoing leg; translated into the CTP framework and for the vertex with the photon on the outgoing leg their result reads:

$$
\left(p^{\prime}-p\right)_{\mu} G_{a b c}^{\mu}\left(p,-p^{\prime}, p^{\prime}-p\right)=-i g\left[\delta_{b c} \Sigma_{a c}\left(p^{\prime}\right)-\delta_{a c} \Sigma_{c b}(p)\right]
$$

where $a, b, c=1,2$. This can be rewritten in terms of the retarded and advanced amplitudes; the result agrees with what we find for the HTL amplitudes below.

In order to examine the relations among the 2- and 3-point HTLs in the real time formalism, we calculate the retarded electron self-energy [11:

$$
\Sigma_{R}(p)=g^{2} \int \frac{d^{3} \boldsymbol{k}}{(2 \pi)^{3}} \int d \omega d \omega^{\prime} \rho_{f}(\omega, \boldsymbol{p}+\boldsymbol{k}) \rho_{B}\left(\omega^{\prime}, \boldsymbol{k}\right)\left(\frac{n\left(\omega^{\prime}\right)+\tilde{n}(\omega)}{p_{0}+\omega^{\prime}-\omega+i \epsilon}\right)
$$


where $\rho_{F}$ and $\rho_{B}$ are the two-point spectral densities of the fermions and bosons, respectively. Inserting the free particle spectral densities

$$
\begin{aligned}
\rho_{B}\left(\omega^{\prime}, \boldsymbol{k}\right) & =\operatorname{sgn}\left(\omega^{\prime}\right) \delta\left(\omega^{\prime 2}-E_{k}^{2}\right), \\
\rho_{F}(\omega, \boldsymbol{p}+\boldsymbol{k}) & =\left(\omega \gamma^{0}+\boldsymbol{k}^{\prime} \cdot \boldsymbol{\gamma}+m\right) \operatorname{sgn}(\omega) \delta\left(\omega^{2}-E_{p+k}^{2}\right),
\end{aligned}
$$

with $E_{k}=\sqrt{\boldsymbol{k}^{2}}, E_{k}^{\prime}=\sqrt{\boldsymbol{k}^{2}+m^{2}}, k^{\prime}=k+p$, and evaluating the integral at in the HTL approximation and using dimensional regularization [32], we obtain

$$
\Sigma_{R}(p)=a(p) \not p+b(p) \gamma_{0}
$$

where

$$
\begin{aligned}
& a\left(p_{0}, \boldsymbol{p}\right)=\frac{m_{\beta}^{2}}{\boldsymbol{p}^{2}}\left(1-\frac{p_{0}}{4 \pi} \int d \Omega \frac{1}{V_{+} \cdot p+i \epsilon}\right) \\
& b\left(p_{0}, \boldsymbol{p}\right)=\frac{m_{\beta}^{2}}{\boldsymbol{p}^{2}}\left(p_{0}+\frac{p^{2}}{4 \pi} \int d \Omega \frac{1}{V_{+} \cdot p+i \epsilon}\right) .
\end{aligned}
$$

Here $V_{+}=(1, \boldsymbol{V}), \boldsymbol{V}=\frac{\boldsymbol{k}}{|\boldsymbol{k}|}$, and the integration is over the direction of the unit vector $\boldsymbol{V}$. Some further algebra then leads directly to

$$
\Sigma_{R}(p)=\frac{m_{\beta}^{2}}{4 \pi} \int d \Omega \frac{V_{+}}{V_{+} \cdot p+i \epsilon} .
$$

The advanced electron self-energy in HTL approximation is computed similarly as

$$
\Sigma_{A}(p)=\frac{m_{\beta}^{2}}{4 \pi} \int d \Omega \frac{V_{+}}{V_{+} \cdot p^{\prime}-i \epsilon} .
$$

From this one obtains

$$
\begin{aligned}
& \Sigma_{R}\left(p^{\prime}\right)-\Sigma_{R}(p)=-\frac{m_{\beta}^{2}}{4 \pi} \int d \Omega \frac{V_{+} \cdot\left(p^{\prime}-p\right) V_{+}}{\left(V_{+} \cdot p+i \epsilon\right)\left(V_{+} \cdot p^{\prime}+i \epsilon\right)}, \\
& \Sigma_{R}\left(p^{\prime}\right)-\Sigma_{A}(p)=-\frac{m_{\beta}^{2}}{4 \pi} \int d \Omega \frac{V_{+} \cdot\left(p^{\prime}-p\right) V_{+}}{\left(V_{+} \cdot p-i \epsilon\right)\left(V_{+} \cdot p^{\prime}+i \epsilon\right)} . \\
& \Sigma_{A}\left(p^{\prime}\right)-\Sigma_{A}(p)=-\frac{m_{\beta}^{2}}{4 \pi} \int d \Omega \frac{V_{+} \cdot\left(p^{\prime}-p\right) V_{+}}{\left(V_{+} \cdot p-i \epsilon\right)\left(V_{+} \cdot p^{\prime}-i \epsilon\right)} .
\end{aligned}
$$

If we set in (26) $k_{1}=p, k_{2}=-p^{\prime}, k_{3}=p^{\prime}-p$ and then compare it with Eqs. (38) we find the following relations between the hard thermal loop contributions to the 2- and 3-point functions: 


$$
\begin{aligned}
\left(p^{\prime}-p\right)_{\mu} G_{R i}^{\mu}\left(p,-p^{\prime}, p^{\prime}-p\right) & =-i g\left[\Sigma_{R}\left(p^{\prime}\right)-\Sigma_{R}(p)\right], \\
\left(p^{\prime}-p\right)_{\mu} G_{R}^{\mu}\left(p,-p^{\prime}, p^{\prime}-p\right) & =-i g\left[\Sigma_{A}\left(p^{\prime}\right)-\Sigma_{A}(p)\right], \\
\left(p^{\prime}-p\right)^{\mu} G_{R o}^{\mu *}\left(p,-p^{\prime}, p^{\prime}-p\right) & =-i g\left[\Sigma_{A}\left(p^{\prime}\right)-\Sigma_{R}(p)\right] .
\end{aligned}
$$

These relations are structurally similar to the zero temperature Ward identity (30) and agree (up to notational differences) with the general finite temperature Ward identities in RTF given in Eq. (3.17) of Ref. [28].

\section{CONCLUSIONS}

We studied the QED 3-point vertex function at finite temperature in the CTP real-time formalism. This formalism has recently gained increased popularity because it allows for a generalization to non-equilibrium situations as encountered, e.g., in the initial stages of heavy-ion collisions, and it avoids the need for analytical continuation which plagues the imaginary time formalism. We started by giving a set of useful relations among the eight thermal components of the real-time vertex function. We then derived spectral integral representations for the three retarded 3-point functions and calculated the corresponding spectral densities explicitly at 1-loop order in the HTL approximation. In this approximation the two independent spectral densities become degenerate and are transverse with respect to all three external momenta.

Inserting these HTL spectral densities into the spectral representation we obtained three retarded 3-point vertex functions, two of which turned out to be identical to the corresponding QCD-analogues derived in [23] from a set of classical kinetic equations in the long-wavelength limit. By contracting the three 3-point vertex functions with the momentum vector of the photon, we obtained a result which we could compare with the fermion HTL self energy. The result was a set of real-time Ward-Takahashi identities at finite temperature which generalize the zero temperature Ward identity and agree with the recently derived finite temperature identities of Ref. [28. 
Due to the matrix structure of the real-time thermal Green functions, there is a whole class of finite temperature Ward identities which relate retarded and advanced vertex functions to combinations of retarded and advanced fermion self energies. As first observed by D'Olivo et al. [28], if the ingoing (outgoing) fermion leg has the largest time, the Ward identity involves only the retarded (advanced) fermion self energies; if the photon leg has the largest time, both retarded and advanced fermion self energies are involved.

Clearly, no fundamentally new physical results were derived in this paper; this was not our goal. What we have achieved is a consistent real-time representation of the 2- and 3-point functions in finite temperature field theory, taking into account the full matrix structure arising from the doubling of degrees of freedom, which makes their analytic structure explicit and can thus serve as a basis for non-perturbative resummation schemes at finite temperature. We have tested the consistency of the formalism in the context of HTL resummation within thermal equilibrium QED. We expect the formalism to provide a stable basis for an extension to the real-time dynamics of non-equilibrium systems.

\section{ACKNOWLEDGMENTS}

This work was supported by the Deutsche Forschungsgemeinschaft (DFG), the Bundesministerium für Bildung und Forschung (BMBF), the National Natural Science Foundation of China (NSFC), and the Gesellschaft für Schwerionenforschung (GSI). 


\section{REFERENCES}

$\dagger$ e-mail address: defu.hou@rphs1.physik.uni-regensburg.de . On leave of absence from Hua-Zhong Normal University, Wuhan, China

[1] N.P. Landsman and Ch.G. van Weert, Phys. Rep. 145, 141 (1987).

[2] J.I. Kapusta, Finite Temperature Field Theory (Cambridge University Press, 1989).

[3] J.P. Blaizot, J.-Y. Ollitrault, and E. Iancu, in Quark-Gluon Plasma 2, p. 135, ed. by R.C. Hwa (World Scientific, Singapore, 1996).

[4] M.H. Thoma, in Quark-Gluon Plasma 2, p. 51, ed. by R.C. Hwa (World Scientific, Singapore, 1996).

[5] M. LeBellac, Thermal Field Theory (Cambridge University Press, 1996).

[6] S. Jeon, Phys. Rev. D 47, 4586 (1993); and 52, 3591 (1995).

[7] R. Kobes and G.W. Semenoff, Nucl.Phys. B 260, 714 (1985); and 272, 329 (1986).

[8] Hou Defu, S. Ochs, and Li Jiarong, Phys. Rev. D 54, 7634 (1996).

[9] E. Wang and U. Heinz, Phys. Rev. D 53, 899 (1996).

[10] K.-C. Chou, Z.-B. Su, B.-L. Hao, and L. Yu, Phys. Rep. 118, 1 (1985).

[11] P.A. Henning, Phys. Rep. 253, 235 (1995).

[12] P.V. Landshoff, Cambridge University preprint DAMTP 96/64

[13] J.C. Taylor, Phys. Rev. D 48, 958 (1993).

[14] E. Braaten and R.D. Pisarski, Nucl.Phys. B 337, 569 (1990); and 339, 310 (1990).

[15] R. Kobes, Phys. Rev. D 43, 1269 (1991).

[16] T.S. Evans, Phys. Lett. B 249, 286 (1990); 252, 108 (1990); and Nucl. Phys. B 374, $340(1992)$. 
[17] M.E. Carrington and U. Heinz, Eur. Phys. J. C (1998), in press (Los Alamos eprint archive hep-th/9606055).

[18] Hou Defu and U. Heinz, Eur. Phys. J. C (1998), in press (Los Alamos eprint archive hep-th/9704392).

[19] E. Braaten and R.D. Pisarski, Phys. Rev. D 45, R1827 (1992).

[20] J. Frank and J.C. Taylor, Nucl. Phys. B 334, 199 (1990).

[21] J.C.Taylor and S.M.H. Wong, Nucl. Phys. B 346, 115 (1990).

[22] R. Jackiw, V.P. Nair, Phys. Rev. D 48, 4991 (1993).

[23] J.-P. Blaizot and E. Iancu, Phys. Rev. Lett. 70, 3376 (1993); Nucl. Phys. B417, 608 (1994).

[24] P.F. Kelly, Q. Liu, C. Lucchesi, and C. Manuel, Phys. Rev. Lett. 72, 3461 (1994).

[25] J. Frenkel and J.C. Taylor, Nucl.Phys. B 374, 156 (1992).

[26] U. Heinz, Ann. Phys. (N.Y.) 168, 148 (1986).

[27] M.E. Carrington, Hou Defu and M.H. Thoma, Los Alamos eprint archive hep$\mathrm{ph} / 9708363$.

[28] J.C. D’Olivo, M. Torres, and E. Tútuti, Phys. Rev. D 55, 3859 (1997).

[29] J.C. Ward, Phys. Rev. 77, 293 (1950); Y. Takahashi, Nuovo Cimento 6, 340 (1957).

[30] R. Kobes, Phys. Rev. D 42, 562 (1990).

[31] R. Baier, B. Pire, and D. Schiff, Z. Phys. C 51, 581 (1991); P. Aurenche, E. Petitgirard and T.R. Gaztelurrutia, Phys. Lett. B 297, 337 (1992).

[32] H.A. Weldon, Phys. Rev. D 26, 1394 (1982). 